PROCEEDINGS OF THE

AMERICAN MATHEMATICAL SOCIETY

Volume 134, Number 6, Pages 1777-1782

S 0002-9939(05)08183-9

Article electronically published on December 15, 2005

\title{
FRACTIONAL POWERS OF THE ALGEBRAIC SUM OF NORMAL OPERATORS
}

\author{
TOKA DIAGANA
}

(Communicated by Joseph A. Ball)

\begin{abstract}
The main concern in this paper is to give sufficient conditions such that if $A, B$ are unbounded normal operators on a (complex) Hilbert space $\mathbb{H}$, then for each $\alpha \in(0,1)$, the domain $D\left((\overline{A+B})^{\alpha}\right)$ equals $D\left(A^{\alpha}\right) \cap D\left(B^{\alpha}\right)$. It is then verified that such a result can be applied to characterize the domains of fractional powers of a large class of the Hamiltonians with singular potentials arising in quantum mechanics through the study of the Schrödinger equation.
\end{abstract}

\section{INTRODUCTION}

Let $\mathbb{H}$ be a complex Hilbert space with inner product $\langle\cdot, \cdot\rangle$. The main concern in this paper is to give sufficient conditions such that if $A, B$ are (unbounded) normal linear operators on $\mathbb{H}$, then for each $\alpha \in(0,1)$,

$$
D\left((\overline{A+B})^{\alpha}\right)=D\left(A^{\alpha}\right) \cap D\left(B^{\alpha}\right)=D\left((\overline{A+B})^{* \alpha}\right),
$$

where $\overline{A+B}$ denotes the closure of the algebraic sum $A+B$ of $A$ and $B$.

It is then verified that the main result (Theorem 2.1) can be applied to characterize the domains of fractional powers of a large class of the Hamiltonians with singular complex potentials arising in quantum mechanics through the study of the well-known Schrödinger equation. For more on these and related issues, see 1 and 2. More generally, note that Theorem 2.1 is applicable to several other situations, in particular, if the sum $\overline{A+B}$ arises as a general elliptic partial differential operator with homogeneous Dirichlet boundary conditions over some specific domains; see, e.g., Yagi [13].

To deal with the above-mentioned issues, one uses the work of de Bivar-Weinholtz and Lapidus [1] on unbounded normal operators and their applications as well as the author's recent papers on the so-called square root problem of Kato for the algebraic sum of operators; see [3] and [6]. Let us recall that the square root problem of Kato for the sum $\overline{A+B}$ amounts to showing that the domain of the square root $\overline{A+B}^{\frac{1}{2}}$ of $\overline{A+B}$ coincides with the domain of its adjoint $\overline{A+B}^{* \frac{1}{2}}$.

Let $A$ be an unbounded normal linear operator on $\mathbb{H}$ (i.e., $A$ is a densely defined closed linear operator on $\mathbb{H}$ such that $A A^{*}=A^{*} A$ ). Using the spectral theorem for

Received by the editors July 12, 2004 and, in revised form, January 31, 2005.

2000 Mathematics Subject Classification. Primary 47B44, 47B25, 47B15.

Key words and phrases. Normal operator, self-adjoint operator, nonnegative operator, fractional powers of operators, algebraic sum, form sum, Hamiltonian, singular potentials. 
unbounded normal operators, one can write

$$
A=A_{1}-i A_{2},
$$

with $A_{1}, A_{2}$ (possibly unbounded) commuting self-adjoint linear operators on $\mathbb{H}$, where $A_{1}, A_{2}$ are respectively the real and the imaginary parts of $A$. Moreover, if $A_{1}, A_{2}$ are nonnegative, then $i A$ is m-accretive; see, e.g., [1] ( $i$ being the imaginary complex number such that $i^{2}=-1$ ).

Recall that if $A, B$ are normal linear operators on $\mathbb{H}$, then their algebraic sum $A+B$ is defined by $D(A+B)=D(A) \cap D(B)$, and $(A+B) u:=A u+B u$ for each $u \in D(A) \cap D(B)$.

For each $\alpha \in(0,1)$, let $A^{\alpha}$ and $B^{\alpha}$ denote the $\alpha$ th powers of $A$ and $B$, respectively; see, e.g., [8]. Observe that both $A^{\alpha}$ and $B^{\alpha}$ are defined by use of the spectral theorem for unbounded normal operators. Furthermore, for each $\alpha \in[0,1]$, the $\alpha$ th interpolation space between $D(A)$ and $\mathbb{H}$ is defined by

$$
D\left(A^{\alpha}\right):=[D(A), \mathbb{H}]_{\alpha} .
$$

Similarly, $D\left(B^{\alpha}\right)=[D(B), \mathbb{H}]_{\alpha}$. For more on interpolation spaces and related issues, we refer the reader to $[8,9,10,13$ and the references therein. In this way, if one supposes that the sum $A+B$ of $A$ and $B$ is nontrivial, i.e., $D(A) \cap D(B) \neq\{0\}$, then the domain of the fractional power $(A+B)^{\alpha}$ of $A+B$ is defined by

$$
D\left((A+B)^{\alpha}\right)=[D(A) \cap D(B), \mathbb{H}]_{\alpha}, \text { for each } \alpha \in[0,1] .
$$

It should be observed that for normal linear operators $A$ and $B, D(A)=D(|A|)$ and $D(B)=D(|B|)$, where $|A|=\left(A^{*} A\right)^{\frac{1}{2}}$ and $|B|=\left(B^{*} B\right)^{\frac{1}{2}}$. Hence,

$$
\begin{gathered}
D\left(A^{\alpha}\right)=D\left(|A|^{\alpha}\right)=D\left(A_{1}^{\alpha}\right) \cap D\left(A_{2}^{\alpha}\right) \text { and } \\
D\left(B^{\alpha}\right)=D\left(|B|^{\alpha}\right)=D\left(B_{1}^{\alpha}\right) \cap D\left(B_{2}^{\alpha}\right),
\end{gathered}
$$

with

$$
A=A_{1}-i A_{2}, \quad B=B_{1}-i B_{2},
$$

where $A_{j}, B_{j}(j=1,2)$ are respectively self-adjoint operators on $\mathbb{H}$.

The paper is organized as follows: in section 2, it will be shown that under some additional assumptions, (1) holds (Theorem 2.1).

Section 3 provides an application of Theorem 2.1 to a large class of the Hamiltonians with singular complex potentials.

Throughout the rest of the paper, $\mathbb{H}, A$ and $B$, and $A+B$ stand for a complex Hilbert space with inner product $\langle\cdot, \cdot\rangle$, unbounded normal linear operators on $\mathbb{H}$, and the algebraic sum of $A$ and $B$, respectively.

In applications, especially those arising in quantum mechanics, it is more convenient to choose the potential so that the negative part of its real part is not too large in sense of quadratic forms. To achieve such a goal, one replaces an assumption such as " $B_{1}$ nonnegative" by a weaker assumption on its nonnegative part. For that, using the spectral theorem, one can write

$$
B_{1}=B_{+}-B_{-},
$$

where $B_{+}, B_{-}$are respectively the positive and negative parts of the real part $B_{1}$ of $B$. Next, one supposes that $B_{-}$is relatively a form bounded with respect to $A_{1}$ with bound $<1$; see (H.3) below. 
In view of the above, for each $0<\alpha<1$, one sets $K_{\alpha}=K\left(A^{\alpha}\right) \cap K\left(B^{\alpha}\right)$, where

$$
K\left(A^{\alpha}\right):=D\left(|A|^{\alpha}\right)=D\left(A_{1}^{\alpha}\right) \cap D\left(A_{2}^{\alpha}\right)
$$

and

$$
K\left(B^{\alpha}\right):=D\left(|B|^{\alpha}\right)=D\left(\left(B_{+}\right)^{\alpha}\right) \cap D\left(\left(B_{-}\right)^{\alpha}\right) \cap D\left(B_{2}^{\alpha}\right) .
$$

Throughout the rest of the paper, one supposes that $A=A_{1}-i A_{2}$ and $B=$ $B_{1}-i B_{2}$ are normal operators such that $A+B$ is densely defined and such that

(H.1) there exists $\mu>0$ such that $\left|\left\langle A_{1} u, u\right\rangle\right| \leq \mu\left\langle A_{2} u, u\right\rangle, \forall u \in K\left(A^{\frac{1}{2}}\right)$;

(H.2) there exists $\nu>0$ such that $\left|\left\langle B_{1} u, u\right\rangle\right| \leq \nu\left\langle B_{2} u, u\right\rangle, \quad \forall u \in K\left(B^{\frac{1}{2}}\right)$;

(H.3) $D\left(A_{1}^{\frac{1}{2}}\right) \subset D\left(B_{-}^{\frac{1}{2}}\right)$, and there exist positive constants $\gamma<1$ and $\delta$ such that

$$
\left\|B_{-}^{\frac{1}{2}} u\right\|^{2} \leq \gamma\left\|A_{1}^{\frac{1}{2}} u\right\|^{2}+\delta\|u\|^{2}, \quad \forall u \in D\left(A_{1}^{\frac{1}{2}}\right)
$$

If $\phi, \psi$ are respectively the sesquilinear forms associated with $A$ and $B$, then one can write

$$
\begin{gathered}
\phi(u, v)=\left\langle A_{1}^{\frac{1}{2}} u, A_{1}^{\frac{1}{2}} v\right\rangle-i\left\langle A_{2}^{\frac{1}{2}} u, A_{2}^{\frac{1}{2}} v\right\rangle, \quad \forall u, v \in K\left(A^{\frac{1}{2}}\right), \quad \text { and } \\
\psi(u, v)=\left\langle B_{+}^{\frac{1}{2}} u, B_{+}^{\frac{1}{2}} v\right\rangle-\left\langle B_{-}^{\frac{1}{2}} u, B_{-}^{\frac{1}{2}} v\right\rangle-i\left\langle B_{2}^{\frac{1}{2}} u, B_{2}^{\frac{1}{2}} v\right\rangle, \quad \forall u, v \in K\left(B^{\frac{1}{2}}\right) .
\end{gathered}
$$

Now set, $\xi(u, v)=\phi(u, v)+\psi(u, v)$, for all $u, v \in K_{\frac{1}{2}}$.

According to de Bivar-Weinholtz and Lapidus [1, p. 451], the "Generalized" sum (or form sum) $A \dot{+} B$ of $A$ and $B$ is defined with the help of the sesquilinear form $\xi$ as follows: $u \in D(A \dot{+} B)$ if and only if the mapping $v \longrightarrow \xi(u, v)$ is continuous for the $\mathbb{H}$-topology, and that $(A \dot{+} B) u$ is defined to be the vector of $\mathbb{H}$ given by the Riesz representation theorem

$$
\langle(A+B) u, v\rangle=\xi(u, v), \quad u \in D(A+B), \quad \forall v \in K_{\frac{1}{2}} .
$$

Consequently, if $A+B$ is densely defined, then $\xi$ has the following representation: $\xi(u, v)=\langle(A+B) u, v\rangle$, for all $u \in D(A) \cap D(B), \forall v \in K_{\frac{1}{2}}$.

\section{Fractional POWERS OF OPERATORS}

Theorem 2.1. Let $A=A_{1}-i A_{2}, B=B_{1}-i B_{2}$ be (unbounded) normal linear operators on $\mathbb{H}$. Under previous assumptions, suppose that (H.1), (H.2), and (H.3) hold and that $\overline{i(A+B)}$ is maximal. Then,

$$
D\left((\overline{A+B})^{\alpha}\right)=D\left(A^{\alpha}\right) \cap D\left(B^{\alpha}\right)=D\left((\overline{A+B})^{* \alpha}\right)
$$

for each $0<\alpha<1$.

Proof. From (4), it is clear that the form sum $A \dot{+} B$ is the operator associated with the sesquilinear form $\xi$. It is also well known [1, p. 451] that the operator $i(A \dot{+} B)$ is m-accretive, by (H.3). Define the sesquilinear form $\zeta$ by setting

$$
\zeta(u, v):=\langle i(A+B) u, v\rangle, \quad u \in D(A+B), \quad \forall v \in K_{\frac{1}{2}} .
$$

It is then clear that $\zeta$ is a densely defined closed sectorial sesquilinear form, by (H.2), (H.3), and the facts above. Now since both $\overline{i(A+B)}$ and $i(A \dot{+} B)$ are maximal extensions of $A+B$, it follows that $\overline{i(A+B)}=i(A+B)$, and hence $\overline{i(A+B)}$ is the m-sectorial operator associated with the sesquilinear form $\zeta$.

We first consider the critical case $\alpha=\frac{1}{2}$. Since $D(A)=D\left(A^{*}\right)$ and $D(B)=$ $D\left(B^{*}\right)$ it follows that $D(\overline{i(A+B)}) \subset D\left((\overline{i(A+B)})^{*}\right)$, that is,

$$
D\left(\overline{A+B} \subset D\left((\overline{A+B})^{*}\right),\right.
$$


and hence, by Lions [10, Theorem 5.2, p. 238],

$$
D\left((\overline{A+B})^{\frac{1}{2}}\right) \subset D(\zeta)=K_{\frac{1}{2}} \subset D\left((\overline{A+B})^{* \frac{1}{2}}\right) .
$$

Similarly, considering the conjugate $\zeta^{*}$ of $\zeta$ with $D(\zeta)=D\left(\zeta^{*}\right)$, and using similar arguments as above for the adjoint $\overline{i(A+B)}$ * of $\overline{i(A+B)}$, it follows that

$$
D\left((\overline{i(A+B)})^{* \frac{1}{2}}\right) \subset D\left((\overline{i(A+B)})^{\frac{1}{2}}\right),
$$

that is,

$$
D\left((\overline{A+B})^{* \frac{1}{2}}\right) \subset D\left((\overline{A+B})^{\frac{1}{2}}\right) .
$$

Again, by Lions [10, Theorem 5.2, p. 238],

$$
D\left((\overline{A+B})^{* \frac{1}{2}}\right) \subset K_{\frac{1}{2}} \subset D\left((\overline{A+B})^{\frac{1}{2}}\right) .
$$

In view of (6) and (8) it follows that

$$
D\left((\overline{A+B})^{\frac{1}{2}}\right)=D\left(A^{\frac{1}{2}}\right) \cap D\left(B^{\frac{1}{2}}\right)=D\left((\overline{A+B})^{* \frac{1}{2}}\right) .
$$

It remains the noncritical case $\alpha \in\left(0, \frac{1}{2}\right) \cup\left(\frac{1}{2}, 1\right)$. For that, one follows along the same line as in the proof for the critical case $\alpha=\frac{1}{2}$. Indeed, from (5), Lions [10, Theorem 5.2, p. 238], and Kato [8] it follows that

$$
D\left((\overline{A+B})^{\alpha}\right) \subset K_{\alpha} \subset D\left((\overline{A+B})^{* \alpha}\right), \quad \forall \alpha \in\left(0, \frac{1}{2}\right) \cup\left(\frac{1}{2}, 1\right) .
$$

Similarly,

$$
D\left((\overline{A+B})^{* \alpha}\right) \subset K_{\alpha} \subset D\left((\overline{A+B})^{\alpha}\right), \quad \forall \alpha \in\left(0, \frac{1}{2}\right) \cup\left(\frac{1}{2}, 1\right) .
$$

Combining (9) and (10), one obtains that

$$
D\left((\overline{A+B})^{\alpha}\right)=D\left(A^{\alpha}\right) \cap D\left(B^{\alpha}\right)=D\left((\overline{A+B})^{* \alpha}\right), \quad \forall \alpha \in\left(0, \frac{1}{2}\right) \cup\left(\frac{1}{2}, 1\right) .
$$

We then conclude that

$$
D\left((\overline{A+B})^{\alpha}\right)=D\left(A^{\alpha}\right) \cap D\left(B^{\alpha}\right)=D\left((\overline{A+B})^{* \alpha}\right), \quad \forall \alpha \in(0,1) .
$$

Remark 2.2. Let us note that if one omits both (H.1) and (H.2), then Theorem 2.1 still holds for each $\alpha \in\left(0, \frac{1}{2}\right) \cup\left(\frac{1}{2}, 1\right)$; see Kato [8] for details.

\section{Applications}

This section provides an application of Theorem 2.1 related to the characterization of the domains of fractional powers of the so-called Hamiltonians with singular potentials studied by Brézis and Kato [2] and de Bivar-Weinholtz and Lapidus [1].

Throughout this section, we suppose $\mathbb{H}=L^{2}\left(\mathbb{R}^{d}\right)$ is equipped with its natural inner product. Let $\Phi$ be the sesquilinear form defined by

$$
\Phi(u, v)=\int_{\mathbb{R}^{d}} \nabla u \overline{\nabla v} d x, \quad \forall u, v \in D(\Phi)=\mathbb{H}^{1}\left(\mathbb{R}^{d}\right) .
$$

Clearly, $\Phi$ is a sectorial sesquilinear form with domain $D(\Phi)=\mathbb{H}^{1}\left(\mathbb{R}^{d}\right)$. On the other hand, let $\Psi$ be the sesquilinear form defined by

$$
\Psi(u, v)=\int_{\mathbb{R}^{d}} q u \bar{v} d x, \quad \forall u, v \in D(\Psi),
$$


with $q=q^{+}-q^{-}-i q^{\prime}$, where $q^{+}, q^{-}, q^{\prime} \in L_{l o c}^{1}\left(\mathbb{R}^{d}\right)$ are real nonnegative functions, and

$$
D(\Psi)=\left\{u \in L^{2}\left(\mathbb{R}^{d}\right): \int_{\mathbb{R}^{d}}|q(x)| .|u(x)|^{2} d x<\infty\right\} .
$$

In view of the above, it is not hard to see that the operators associated with $\Phi, \Psi$ are respectively defined by

$$
\begin{gathered}
D(A)=\mathbb{H}^{2}\left(\mathbb{R}^{d}\right), \quad A u=-\Delta u, \text { for each } u \in D(A), \text { and } \\
D(B)=\left\{u \in L^{2}\left(\mathbb{R}^{d}\right):\left(q^{+}-q^{-}-i q^{\prime}\right) u \in L^{2}\left(\mathbb{R}^{d}\right)\right\}, \quad B u=\left(q^{+}-q^{-}-i q^{\prime}\right) u
\end{gathered}
$$

for each $u \in D(B)$, where $\Delta$ denotes the $d$-dimensional Laplace operator.

It is also routine to check that both $A$ and $B$ are unbounded normal operators on $L^{2}\left(\mathbb{R}^{d}\right)$. Now $A=A_{1}-i A_{2}$, and $B=B_{1}-i B_{2}$, where $A_{1}=0, A_{2}=-\Delta$, $B_{1}=q^{+}-q^{-}$, and $B_{2}=q^{\prime}$ are nonnegative self-adjoint operators, respectively $\left(B_{1}, B_{2}\right.$ being the maximal multiplication operators associated with the potentials $q^{+}-q^{-}$and $q^{\prime}$, respectively).

Let us note that since $A_{1}=0$, the assumption (H.3) would impose that $q_{-}$is relatively bounded. In this event, it is sufficient to suppose only that $q_{-} \in L_{l o c}^{1}\left(\mathbb{R}^{d}\right)$; see details in [1].

Let $\Xi=\Phi+\Psi$. It is well known [1] that the form sum $A \dot{+} B$ associated with the sesquilinear form $\Xi$ coincides with the natural realization $\mathcal{S}$ of $i \Delta+q$ defined by

$$
D(\mathcal{S})=\left\{u \in \mathbb{H}^{1}\left(\mathbb{R}^{d}\right): q u \in L_{l o c}^{1}\left(\mathbb{R}^{d}\right) \text { and } i \Delta u+q u \in L^{2}\left(\mathbb{R}^{d}\right)\right\}
$$

and

$$
\mathcal{S} u=i \Delta u+q u, \quad \forall u \in D(\mathcal{S}) .
$$

Now from (H.1), (H.2), and the previous facts, $\overline{i(i \Delta+q)}=\overline{-\Delta+i q}$ is maximal; see, Brézis and Kato [2]. Therefore,

$$
D\left((\overline{i \Delta+q})^{\alpha}\right)=\mathbb{H}^{2 \alpha}\left(\mathbb{R}^{d}\right) \cap D\left(B^{\alpha}\right)=D\left((\overline{i \Delta+q})^{* \alpha}\right), \quad \forall \alpha \in(0,1),
$$

by Theorem 2.1 .

\section{ACKNOWLEDGEMENT}

The author expresses his deepest thanks to the referee for comments and significant suggestions on the first version of the paper.

\section{REFERENCES}

1. A. de Bivar-Weinholtz and M. L. Lapidus, Product Formula for Resolvents of Normal Operator and the Modified Feynman Integral. Proc. Amer. Math. Soc. 110 (1990), no. 2, 449-460. MR1013964 (91f:47052)

2. H. Brézis and T. Kato, Remarks on the Schrödinger Operator with Singular Complex Potentials, J. Math. Pures Appl. 58 (1979), 137-151. MR0539217 (80i:35135)

3. T. Diagana, Sommes d'opérateurs et conjecture de Kato-McIntosh, C. R. Acad. Sci. Paris, t. 330, Série I (2000), 461-464. MR.1756959 (2001f:47058)

4. T. Diagana, Variational Sum and Kato's Conjecture, J. Convex Anal. 9 (2002), no. 1, 291-294. MR.1917402 (2003e:47089)

5. T. Diagana, Quelques remarques sur l'opérateur de Schr̈odinger avec un potentiel complexe singulier particulier. Bull. Belgian. Math. Soc. 9 (2002), 293-298. MR2017083 (2004f:47108)

6. T. Diagana, Algebraic Sum of Unbounded Normal Operators and the Square Root Problem of Kato. Rend. Sem. Math. Univ. Padova, 110 (2003), 269-275. MR2033011(2005b:47047)

7. T. Kato, Perturbation theory for linear operators, New York (1966). MR0203473 (34:3324) 
8. T. Kato, Fractional Powers of Dissipative Operators II. J. Math. Soc. Japan. 14 (1962), 242-248. MR0151868 (27:1851)

9. J.-L. Lions, Espace intermédiaires entre espaces Hilbertiens et applications. Bull. Math. Soc. Sci. Math. Phys. R. P. Roumanie (N.S.) 2 (50) (1958), 419-432. MR0151829 (27:1812)

10. J.-L. Lions, Espaces d'interpolation et domaines de puissances fractionnaires d'opérateurs. $J$. Math. Soc. Japan 14 (1962), 233-241. MR0152878 (27:2850)

11. A. Pazy, Semigroups of linear operators and application to partial differential equations, Springer-Verlag, New York (1983). MR.0710486 (85g:47061)

12. W. Rudin, Functional Analysis, McGraw-Hill, New York (1973). MR0365062 (51:1315)

13. A. Yagi, Coincidence entre des espaces d'interpolation et des domaines de puissances fractionnaires d'opérateurs. C. R. Acad. Sci. Paris Sér. I Math. 299 (1984), no. 6, 173-176. MR0759225 (86c:46090)

Department of Mathematics, Howard University, 2441 6th Street N.W., Washington D.C. 20059

E-mail address: tdiagana@howard.edu 